\title{
An Analysis of Personality Adjustment influenced by Early Traumatic Disorder (ETD)
}

Manashi Singha*

Vidyasagar School of Social Work, Psycho-Counselling, India

\begin{abstract}
There frequently a violation happened on fresher entry in different environment and in post marital relationship where person's desire (subconscious concept on individual practice), expectation \& emotion about fundamental behaviour and extreme pleasure and satisfaction become questing on insecurity of Trans-Survival Environment or TSE. Where non-cooperation or refusal on the partner's request for work coordination or safe sex on using nonconsensual force or roughness or unwanted sadistic behavioural or sexual acts, disregard for the counterpart's feelings, aimed exerting control over the partner become a substance abuse that experienced an overwhelming amount of stress. Such an event or unforced situation that causes disruption on relationship and make great distress on personality adjustment or unpleasant experience that causes Early Traumatic Disorder (ETD).
\end{abstract}

Keywords: Pursuance concept; Pro night sleeping syndrome; Cognitive installation; Mind circulations; Post marital traumas; TPA

\section{Introduction}

\section{Objective}

The study of Early Traumatic Disorder (ETD) on personality adjustment is an attempt to assess and differentiate from the general pursuance of Psychological Disorder. The aim of this study is to assess adulthood early developmental trauma (EDT) and Post Marital Adjustment with Social Anxiety Disorder (SAD) under various Psychological factors influence. The object of this study also to be find out mental disbursement setup in psychological motivation on personality adjustment under interchanging environment (intrapersonal and interpersonal relationship) that affected different ageing of personality with substance adjustment. This particular study also emphasis on mind circulation flow and mental cognitive resources analysis which disburse feelings and emotion on optimum tolerance under personality adjustment.

\section{Background}

The theme of this study was an experimental knowledge of clinical diagnoses on different ageing personality with different socioeconomic and educational background where a Participant factor (Client existence) identified as clinical experiment. The Participant was a two month old married, nineteen plus year of ageing female, admitted in a self-financing 'Psychological Rehabilitation Centre'*+, by her in-law family members (husband's sister and two other) for performance development but how and what kind of development that was unknown (unable to expose or making any assumption about the causes, object \& ageing difference) to the participant (client). Before married the participant (client) lived in maternal uncle house with cousins, far away from her Home land (parent's house) for nonconventional marital separation of parent, under a joint family environment since 2 Years of age to marriage. Though it was an arrange marriage but the participant (client) got opportunity to interact and enjoying pre-marital communication regularly over a Year through cell phone with the would- be husband. Participant (client) also got opportunity in making choice after sharing own thinking, feelings and motivation with the counterpart on Viva-Visa along with two different dates on family consent. Participant (client) had passion on writing Shaire (Hindi Poetry) and recite, and Classical Music listening which was appreciated by would be husband. The participant's (client) fast time sexual relation with husband was a good feeling that happened two day after marriage. The Participant (client) also did only two- three time sexual relation with husband before sheltering in Psychological Rehabilitation Centre that was not clearly exposed during counseling.

\section{Assessment}

On the primary assessment it was unclear how the particular marriage relationship becoming speculated on psychological concern. In general, cognitive literacy about marriage life might be helpful for self-assessment of age-related differences and partner contribution and demands in making adjustment on such relationship needed and critical practice. The role of marriage and family performance (Supportive behaviour) and partner's sensible attitude such as holding hands with hugging, looking one another with eye gesturing, personal care on demand etc. within the relationship practices was confirmed by the Participant. It was hard to separate marriage relationship from the above background but it happened.

This particular study on empirical knowledge and interaction was helpful in making interpretation of post-marital relationship under application, initiation, adjustment and enhancement. The participant (client) asserted, "She need some time for adjustment and the counterpart assured". From the provided particular information made evidence of increased level of stress on personality adjustment and accumulate anxiety on relationship (sexual relation) that arouse early mental (Psychological) trauma in stable married life.

The Early Psychological Trauma (EPT) developed whatever caused for emotional, physical and sexual abuse that experienced

*Corresponding author: Manashi Singha, Vidyasagar School of Social Work, Psycho-Counselling, DD 18/4/1, Salt Lake City, Kolkata, West Bengal 700 064, India, Tel: +91- 9477815216; E-mail: singhamanashi@gmail.com

Received June 22, 2014; Accepted January 08, 2015; Published January 11, 2015

Citation: Singha M (2015) An Analysis of Personality Adjustment influenced by Early Traumatic Disorder (ETD). J Trauma Treat 4: 235. doi: 10.4172/2167-1222.1000235

Copyright: (c) 2015 Singha M. This is an open-access article distributed under the terms of the Creative Commons Attribution License, which permits unrestricted use, distribution, and reproduction in any medium, provided the original author and source are credited. 
overwhelming had been hypothesized to play a significant role in the development of anxiety disorders, such as Substance Stress Disorder (SSD). Eventually this SSD gradually transformed multiple frightening behavioral attitudes like Pro-Night Sleeping Syndrome* (PNSS), passive aggressions on intra-personal close relationship (IPCR) for instanceboldly avoid or escape from one to one personal space or physical intimacy (intercourse), melancholia (inactive attitude) etc. Which became difficult on maintaining post marital personality adjustment (PMPA) under anxiety feeling?

\section{Method}

In this study used clinical data obtained from individual Case History on response and interaction. Personal experience that examined individual personality and personality development by Early Developmental Trauma Questionnaire (EDTQ) on variance was performed. And its Analysis on variance was performed to assess differences. Correlations of answer Questionnaire) were calculated between pre-entry level cognitive installation and post entry environmental (Post Marital Traumas or PMT) effect on thinking, feeling and experiences under stimulation, provocation and justification with the help of participant (client). Most important method that induced to the participant (client) was stirring self-awareness with focusing relationship under self-capability and capacity tolerance with flexible analysis to find out self-belief on substance adjustment.

\section{Analysis}

On innovative efficiency, Human beings are the most developed comparing others species. And Human personality developed with the Pursuance Concept (P C) of society, culture \& custom, religion \& relationship differently and discriminately. As to Human beings are more dedicated on individual means (desire, expectation, requirements, consumptions and satisfaction) and Human's Individualism Psychology (H I P).

This H I P creates different personality with mental discrimination and arises as variance ideology. Moreover, Personality development constantly working in this area aiming balance on complete personality behaviour with the flexibility on TPA.

\section{Now question what is term personality Adjustment (TPA)?}

It is a pattern of behavioural flexibility on extended tolerance of individual's mental disbursement. TPA means individual's mental adaptation capability on interchanging environment whatever it Geographic, functioning, religion and relationship (inter-personal family relationship, intra-cultural social relationship, inter-member functioning relationship and personal marital relationship) under personality development.

Basically individual's adjustment capability influence by three different factors which are directly making impact on personality development and personality motivation. Which are identified as:

\section{(i) Pursuance Concept (P C factor)}

(ii) Cognitive Installation (C I factor)

(iii) Mind Circulation Setup (M C S factor)

You believe or not individual personality developed differently and discriminately depending on these factors influence on mental sensitivities with transformation of knowledge processing and its adaptation on substance situation.

\section{Pursuance concept or P C factors}

"All creatures are idiot on their behaviour". It is an open truth of Psychology that every animal being establishes their existence through pursuing front- runner. As far human beings concern, a particular personality mostly influenced by other Personality who achieve similar goal. What you eat, wear, even your every choice making directly or indirectly influenced by others.

Or it says that your approach whatever it good or bad influenced by others. These happen for our subconscious information which transforming as knowledge processing from mental storage.

As far personality adjustment concern, every individual grown up with a pattern of personality development where individual personality accumulated some forcing degree under the pattern. On later stage it may difficult to re-accumulate under interchanging situation force and the personality compromise with substance force under maximum tolerance.

Moreover these compromises become effective under pursuance pattern of personality behaviour with adjustment on substance situation that also a pursuit of comparable personality.

Eventually personality adjustment is more than a state of personality behaviour under substance situation. It is a Psychological state of personality behaviour under cognitive pursuance factors.

\section{Cognitive installation factor or C I F}

Individual personality development is a super active mechanism with knowledge installation through the ageing. You can identify to those which you have known about or data \& information store in mind through the cognitive installation.

Human Cognitive Installation is a continuing process from born to expiry through various factor resources like Biological Factor Influence (BFI), Environmental Factor Influence (EFI), Substantial Factor Influence (SFI), Heriditical Factor Influence (HFI), Ordinal Position Factor Influence (OFI), Interchanging Mobility Factor Influence (IMFI) etc (Figure 1).

During cognitive installation, your knowledge processing what you have seen, learn or experiencing randomly store in your mind without cleaning or modifying events or situation or its good or bad impact or effectiveness which vastly liable for discriminate personality behaviour and personality adjustment problems.

\section{Mind Circulation Setup factor or M C S factors}

Person's subconscious \& conscious mind create insecureness when sub-conscious factors comparatively enlarge than conscious factors, in repercussion motivation on personality trait and personality development face competitive under mind set up. The data and information storage during cognitive installation develop under mind circulation flow and transform into knowledge processing resource through cognitive influence factors. Personality adjustment depends on how mind disburse knowledge processing resource from mind circulation flow.

Basically Personality adjustment is nothing but a balancing force of behavioural tolerance for survives existence under mind circulation setup (Figure 2).

In general, personality development constructed mental state of analyzing power through the cognitive installation in mind setup. Personality behaviour is a disbursement of mental states from mind 


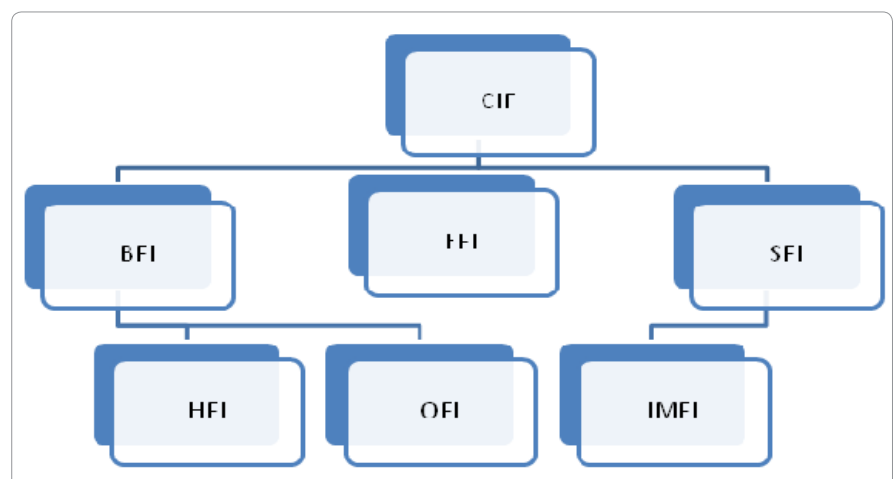

Figure 1: Classification of Cognitive Installation.

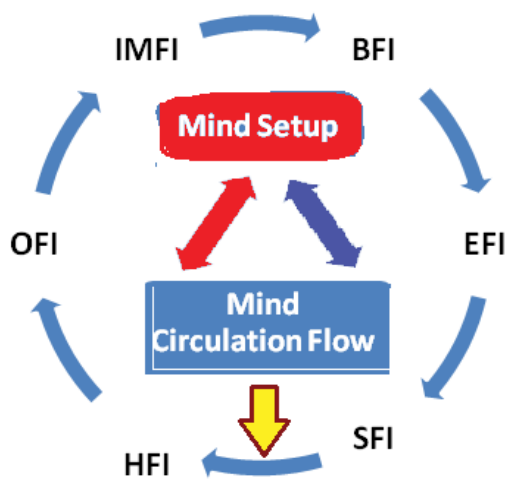

Figure 2: Personality Development in ETD.

circulation flow that make influence and control over personality adjustment under substance environment. But trauma is different mental state of emotional disbursement accumulated from feeling and its optimum analyses with personality development under certain occurrence event or events.

Feeling is a certain analyzing mechanism articulate with emotion, depression, frustration, retaliation and satisfaction. When person cannot analysis feeling properly or unable to analyze feeling on occurrence event or events face mental stress which transform into traumatic disorder.

\section{Result}

There were no significant differences between ETD and PMT under TPA. Primly females were experiencing more PMT along with modern liberal lifestyle comparing counterpart. It occurred because females were emotionally more involved with the sense of cognitive installation pattern.
In this study participant (client) demanding something orthodox as alleviation on anxiety but not exposed in appropriate comments or event about post marital sexual relationship which silently transform into most sensitive and critical stages of psychological development on personality adjustment and cause ETD.

The state resulting was the participant's (client) potentially recovered from the Early Trauma through the process that actually measured on Correlation by exposure of mental exhaustion. It was possible only for the perfect assessment and measured intensity of stress under interchanging situation that was effective to heal developmental trauma by installing self-awareness. on experience.

\section{Conclusion}

The contributions of TPA are important in making extended study on ETD and provide a useful starting point to assess influence over the post marital life and personality adjustment. The opportunity of use DSM-IV structurally limited only under PTSD and it think better to concentrate something different base on need and empirical knowhow under Psychological appreciation.

The pattern introduce and follow in this study with an object that participant's (client) herself able to realize similarity among the causes influence and increased risk on silent exposure of SSD. This study provides evidence of increased levels of multiple types of mental staginess during PMT which are mostly neglected before family relationship performance or use to speculate under Blaming Breakup Relationship (BBR).

However, not every individual exposed too early in response to emotional or behavioural anticipation or challenge in making successful adaptation of Post Marital Relationship (PMR) under anxiety stress. The possibility of this kind of SSD during PMR become increased for various causes like, difference of ageing, conservative cognition installation, physical illness, heriditical background, social \& cultural dissimilarity etc.

Moreover marriage and its practice under family demand mostly enhance emotional biases to new comer where psychosocial problems cannot be excluded from anxiety development under relationship initiation, maintenance, and relationship articulation that become very important on personality adjustment. It may hard task to newly married couple to interface adverse experience.

This Particular study provide strong evidence that early effect of stress silently accumulate adverse experiences therefore it become critical to the victim to assess or understand sources of individual differences in vulnerability to PMPA to ETD (Appendix). 